Pacific Journal of Mathematics

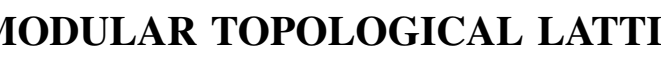




\title{
A MODULAR TOPOLOGICAL LATTICE
}

\author{
Don E. EDMONDSON
}

The purpose of this paper is to present a construction of a compact connected topological lattice which is modular and not distributive. As a special case there will result the example which is a two dimensional subset of $R^{3}$, not embeddable in $R^{2}$.

The existence of such an example is related to structure questions in topological lattices considered by Dyer and Shields [3], Anderson [1], and others.

The first step is to present a general method for constructing a class of modular lattices. Let $D$ denote a distributive lattice which is a chain, $S$ a nonempty set, and $L$ the $S$-fold product lattice of $D$. That is $L=\{f \mid f: S \rightarrow D\}$ and $f \leqq g$ if and only if $f(s) \leqq g(s)$ for every $s \in S$. It is known that $(L, \leqq)$ is a distributive lattice with its operations $\vee$ and $\wedge$ characterized by $[f \vee g](s)=f(s) \vee g(s)$ and

$$
[f \wedge g](s)=f(s) \wedge g(s)
$$

for every $s \in S$. Define

$M=\{f \in L \mid$ there exists $r \in S$ such that $s, t \in S-\{r\}$ implies

$$
f(s) \leqq f(r) \text { and } f(s)=f(t)\} .
$$

For intuition about $M$ and the arguments that follow, note that $M$ simply consists of all of the constant functions of $L$ and the functions of $L$ which are essentially constant in the sense that they assume but two values - the larger value at exactly one point.

If the order of $L, \leqq$, is restricted to $M$, it will be established through a sequence of lemmas that $(M, \leqq)$ is a modular lattice. Recall a lattice $(M, \vee, \wedge)$ is modular if and only if for every $a, b, c \in M$, $b \leqq a$ implies that $a \wedge(b \vee c)=b \vee(a \wedge c)$.

LEMma 1. If $f \in M$ and $f$ is not constant, there exists a unique $r \in S$ such that $s, t \in S-\{r\}$ implies $f(s)<f(r)$ and $f(s)=f(t)$.

The proof of the lemma is immediate from the definition of $M$, and consequently for $f \in M$ and not constant, define index $f$ to be the unique element described in Lemma 1.

Lemma 2. $(M, \leqq)$ is a sub $\wedge$-semilattice of $(L, \leqq)$.

It suffices to show that if $f, g \in M$, then $f \wedge g \in M$. If $f$ and $g$ are 
constant then $f \wedge g$ is constant and therefore in $M$. If $f$ is constant and $g$ is not, let $b=$ index $g$. Then $s, t \in S-\{b\}$ implies

$$
[f \wedge g](s)=f(s) \wedge g(s) \leqq f(s) \wedge g(b)=[f \wedge g](b)
$$

and likewise $[f \wedge g](s)=[f \wedge g](t)$ and thus $f \wedge g \in M$. If $f$ and $g$ $g$ are both not constant, let $a=$ index $f$ and $b=$ index $g$. If $a=b$, then $s, t \in S-\{a\}$ implies $[f \wedge g](s) \leqq[f \wedge g](a)$ and $[f \wedge g](s)=[f \wedge g](t)$. If $a \neq b,[f \wedge g](a)=f(a) \wedge g(a),[f \wedge g](b)=f(b) \wedge g(b)$, and for

$$
x \in S-\{a, b\}[f \wedge g](x)=f(b) \wedge g(a)=[f \wedge g](a) \wedge[f \wedge g](b) .
$$

Then since $D$ is a chain, $[f \wedge g](x)=[f \wedge g](a)$ or $[f \wedge g](x)=[f \wedge g](b)$ depending upon which is minimal and therefore $f \wedge g \in M$.

LEMMA 3. If $a, b, c$ are distinct elements of $S$ and $f \in M$, then $f(a) \wedge f(b)=f(b) \wedge f(c)=f(c) \wedge f(a)$.

The facts of the lemma are an immediate consequence of the definition and is stated as a lemma for convenient reference.

Definition. For $f, g \in M$ define $f \nabla g: S \rightarrow D$ by the following

(i) if $f$ is constant or $g$ is constant, or if $f$ and $g$ are both not constant and index $f=$ index $g$, then $f \nabla g=f \vee g$,

(ii) if $f$ and $g$ are both not constant and index $f \neq$ index $g$, let $a=$ index $f$ and $b=$ index $g$, then

$[f \nabla g](x)=f(x) \vee g(x)$ for $\quad x \in\{a, b\}$

$[f \nabla g](x)=[f(a) \vee g(a)] \wedge[f(b) \vee g(b)]$ for $x \in S-\{a, b\}$.

LEMMA 4. If $f, g \in M$, then

(1) $f \nabla g \in M$ and $f \vee g \leqq f \nabla g$, and

(2) $h \in M, f \leqq h, g \leqq h$ implies $f \nabla g \leqq h$.

In case (i) of the definition of $f \nabla g$, easily $f \nabla g \in M$ and the other results are immediate from $f \nabla g=f \vee g$. In case (ii) let $a=$ index $f$ and $b=$ index $g$, then since $D$ is a chain $[f \nabla g](x)=[f \nabla g](a)$ or $[f \nabla g](x)=[f \nabla g](b)$ for $x \in S-\{a, b\}$. So in this case also $f \nabla g \in M$ and $f \vee g \nabla g$. Also relative to this case, if $h \in M, f \leqq h$, and $g \leqq h$, then $f(x) \vee g(x)=[f \nabla g](x)$ for $x=a$ or $x=b$. But from Lemma 3, $x \in S-\{a, b\}$ implies $h(a) \wedge h(b) \leqq h(x)$ and thus for

$$
x \in S-\{a, b\}[f \nabla g](x)=[f \nabla g](a) \wedge[f \nabla](b) \leqq h(a) \wedge h(b) \leqq h(x) .
$$

Therefore $f \nabla g \leqq h$.

LEMMA 5. If $f, g, h \in M, a, b \in S, a \neq b,[f \vee g](x)=h(x)$ for $x \in\{a, b\}$ 
and $[f \vee g](x) \leqq h(a) \wedge h(b)=h(x)$ for $x \in S-\{a, b\}$, then $h=f \nabla g$.

From the hypothesis $f \vee g \leqq h$ and therefore from Lemma 4 $f \bar{\vee} g \leqq h$. But $h(a)=[f \vee g](a) \leqq[f \nabla g](a)$ and $h(b) \leqq[f \nabla g](b)$. Then from Lemma 3 , for $x \neq a$ and $x \neq b$

$$
h(x)=h(a) \wedge h(b) \leqq[f \nabla g](a) \wedge[f \nabla g](b) \leqq[f \nabla g](x)
$$

and $h \leqq f \nabla g$.

THEOREM 1. $(M, \leqq)$ is a modular lattice with operations $\nabla$ and $\wedge$.

Lemmas 2 and 4 establish that $(M, \leqq)$ is a lattice with operations $\bar{\nabla}$ and $\wedge$, it remains to establish that it is modular. Let $f, g, h \in M$ and $f \leqq g$. It suffices to establish $g \wedge(f \nabla h) \leqq f \nabla(g \wedge h)$ since in any lattice $f \nabla(g \wedge h) \leqq g \wedge(f \nabla)$. The argument will be a case argument.

If $f \nabla h=f \vee h$, then

$$
g \wedge(f \nabla h)=g \wedge(f \vee h)=f \vee(g \wedge h) \leqq f \nabla(g \wedge h)
$$

since $L$ is itself modular and $g \wedge h \in M$ allows Lemma 4 to apply.

If $h \leqq g$, then $f \nabla h \leqq g$ and $g \wedge(f \nabla h)=f \nabla h=f \nabla(g \wedge h)$. If $f \leqq h$, then $f \leqq g \wedge h$ and $g \wedge(f \nabla h)=g \wedge h=f \nabla(g \wedge h)$.

If $f$ and $h$ are not constant, $a=$ index $f, b=$ index $g, a \neq b, h \neq g$, and $f \not h$. Then $f(b)<f(a)$ and $h(a)<h(b)$. Further, $f(a) \leqq h(a)$ implies $f \leqq h$ and therefore $h(a)<f(a)$. Also $h(b) \leqq g(b)$ and $h(a)<f(a) \leqq$ $g(a)$ implies $h \leqq g$ and therefore $h \geqq g$ implies $g(b)<h(b)$. Therefore in this case $h(a)<f(a) \leqq g(a)$ and $f(b) \leqq g(b)<h(b)$. Hence

$$
\begin{aligned}
{[g \wedge(f \nabla h)](a) } & =g(a) \wedge[f(a) \vee h(a)]=f(a) \\
& =f(a) \vee[g(a) \wedge h(a)]=[f \vee(g \wedge h)](a) .
\end{aligned}
$$

Likewise

$$
[g \wedge(f \nabla h)](b)=g(b)=[f \vee(g \wedge h)](b) .
$$

If $x \in S-\{a, b\}$, then

$$
\begin{aligned}
{[g \wedge(f \nabla h)](x) } & =g(x) \wedge[f(a) \vee h(a)] \wedge[f(b) \vee h(b)] \\
& =g(x) \wedge f(a) \wedge h(b)=g(x) \wedge g(a) \wedge f(a) \wedge h(b) \\
& =g(b) \wedge g(a) \wedge f(a) \wedge h(b)=f(a) \wedge g(b) \\
& =[g \wedge(f \nabla h)](a) \wedge[g \wedge(f \nabla h)](b) .
\end{aligned}
$$

But $[f \vee(g \wedge h)](x) \leqq[g \wedge(f \nabla h)](x)$ and $g \wedge(f \nabla h) \in M$, therefore by Lemma $5 g \wedge(f \nabla h)=f \nabla(g \wedge h)$. 
CoRollary. If card $S<3, M$ is a distributive lattice. If $3 \leqq$ card $S$ and $2 \leqq$ card $D$, then $M$ is a modular nondistributive lattice.

If card $S<3$, then $M=L$ and $M$ is distributive. If $3 \leqq$ card $S$ and $2 \leqq$ card $D$, let $s_{1}, s_{2}, s_{3}$ be three distinct elements of $S$ and $c<d$ be two elements of $D$. Define $f_{1}, f_{2}, f_{3}$ by $f_{i}(x)=d$ if $x=s_{i}$ and $f(x)=c$ for $x \in S-\left\{s_{i}\right\}$. Also define $g$ and $k$ by $g(s)=d$ for every $s \in S$ and $k(s)=c$ for every $s \in S$. Then $f_{1} \wedge f_{2}=f_{2} \wedge f_{3} \wedge f_{1}=k$ and $f_{1} \nabla f_{2}=f_{2} \nabla f_{3}=f_{3} \nabla f_{1}=g$ and $\left\{f_{1}, f_{2}, f_{3}, g, k\right\}$ is a modular five sublattice of $M$. Therefore $M$ is not distributive [2].

At this stage the algebraic nature of $M$ has been established, in the section that follows the topological nature of $M$ will be studied. It will be assumed in the following that $D$ is topological chain, that is $D$ is a Hausdorff topological space with the operations $\vee$ and $\wedge$ continuous [3]. If $L$ is considered with the product topology, it is as usual a topological lattice and $M$ may be considered as a topological space in the relative topology that it inherits from $L$. In this context, the following theorem results.

Theorem 2. If $D$ is a topological chain, then

(1) $M$ is a closed subset of $L$,

(2) $M$ is compact if $D$ is compact, and

(3) $M$ is connected if $D$ is connected.

Since with card $S \leqq 2, M=L$, it suffices to consider $3 \leqq$ card $S$ and to establish (1) and (3).

(1) $L-M$ is open for if $f \notin M$, then $f$ is not constant and there exist distinct $a, b, c \in S$ such that $f(b)<f(a)$ and $f(b)<f(c)$. Then since $D$ is a chain $f(b)<f(a) \wedge f(c)$. If there exists $z \in D$ such that $f(b)<z<f(a) \wedge f(c)$, define $W=\{g \in L \mid z<g(a), z<g(c)$, and $g(b)<z\}$ and define $W=\{g \in L \mid f(b)<g(a), f(b)<g(c)$, and $g(b)<f(a) \wedge f(c)\}$ if no such $z$ exists. In either case, $f \in W, W$ is open, and $W \cap M=\varnothing$.

(3) If $D$ is connected, consider the map $T: D \rightarrow M$ where for each $d \in D T(d)=k_{d}$ and $k_{d}$ is the constant function generated by $d$. Clearly $T$ is continuous and $K$ the set of all constant functions is a connected subset of $M$. If $f \in M-K$, let $a=\operatorname{index} \mathrm{f}, m=\max f$, and $r=\min f$ define the map $H$ from $[r, m]=\{x \in D \mid r \leqq x \leqq m\}$ into $M$ by $H(x)=f_{x}$ where $f_{x}(a)=x$ and $f_{x}(s)=r$ for $s \in S-\{a\}$. Again $H$ is continuous and since $[r, m]$ is connected then the range of $H$ is a connected subset of $M$ containing $f$ and intersecting $K$. Therefore $M$ is connected.

Note. It is clear that $\wedge$ will be continuous as an operation on 
$M$ since it is continuous on $L$. Thus when $D$ is a topological chain $M$ is a closed topological sub- $\wedge$-semilattice of $L$. In order to study the operation $\nabla$ relative to continuity, it is necessary to restrict $S$ to being finite, in view of the following lemma.

Lemma 6. If $D$ is a topological chain and $2 \leqq$ card $D$ and $S$ is infinite, then $\bar{\nabla}$ is not continuous.

Let $c<d$ in $D$ and define $k: S \rightarrow D$ by $k(s)=c$ for every $s \in S$. Then $k \nabla k=k$. Let $r \in S$ and define $W_{r}=\{f \in M \mid f(r)<d\}$; then $W_{r}$ is an open subset of $M$ containing $k$. Let $U$ be any open set of $M$ containing $k$, then there exist $s_{1}, s_{2}, \cdots, s_{n}$ distinct elements of $S$ and $U_{1}, U_{2}, \cdots, U_{n}$ open sets of $D$ such that if $W=\left\{f \in M \mid f\left(s_{i}\right) \in U_{i}\right.$ for $i=1,2, \cdots, n\}, k \in W \subset D$. Now $k \in W$ implies

$$
c \in \cap\left\{U_{i} \mid i=1,2, \cdots, n\right\} .
$$

Since $S$ is infinite there exist $a, b \in S-\left\{s_{1}, s_{2}, \cdots, s_{n}\right\}$ such that $a \neq b$. Define $h$ and $g$ by $h(a)=d$ and $h(x)=c$ if $x \in S-\{a\}$, and $g(b)=d$ and $g(x)=c$ if $x \in S-\{b\}$. Therefore $h, g \in W$ and $h \nabla g \notin W_{r}$ since $h \nabla g$ is the constant function defined by $d$. Therefore $U \nabla U \not \subset W_{r}$ and $\bar{V}$ is not continuous.

Definition. For $S$ finite and $2 \leqq$ card $S$, denote max

$$
f=\max \{f(s) \mid s \in S\}, \quad I(f)=\{s \in S \mid f(s)=\max f\} .
$$

Then define $f^{-}: S \rightarrow D$ by

(1) if $I(f)$ is not a unit set, $f^{-}(s)=\max f$ for every $s \in S$, and

(2) if $I(f)$ is a unit set, $f^{-}(s)=\max f$ for $s \in I(f)$, and

$$
f^{-}(s)=\max \{f(t) \mid t \in S-I(f)\} \text { for } s \in S-I(f) .
$$

LEMma 7. If $S$ is finite and $2 \leqq$ card $S$, then

(1) $f \in L$ implies $f^{-} M$ and $f \in M$ if and only if $f=f^{-}$,

(2) $f \leqq f^{-}, f^{--}=f^{-}$, and $f \leqq g$ implies $f^{-} \leqq g^{-}$,

(3) $f, g \in M$ implies $f \nabla g=(f \vee g)^{-}$.

The lemma is a straight forward catalog of the properties following from the definition directly.

Lemma 8. If $S$ is finite, $2 \leqq$ card $S$ and $D$ is a topological chain, then the function $J: L \rightarrow M$ defined by $J(f)=f^{-}$is a retraction of $L$ onto $M$. 
From Lemma 7 it suffices to show that $J$ is continuous. This is done by letting $U$ be an open set in $D$ and $r \in S$, defining $W=$ $\{f \in M \mid f(r) \in U\}$ and showing that $J^{-1}(W)$ is open. It is shown to be open by case argument. Let $g \in L$ and $g^{-} \in W$. If $I(g)$ is not a unit set, then $g^{-}$is constant and $\max g \in U$. Define $V_{1}=\{f \in L \mid f(s) \in U$ for every $s \in S\}$. Since $S$ is finite, $V_{1}$ is open and contains $g$. Further $h \in V_{1}$ implies $h^{-}(r) \in U$. If $I(g)=\{r\}$, let $b$ be an element of $S-\{r\}$. Define $V_{2}=\left\{f \in L \mid f(r) \in U_{1}\right.$, and $f(s) \in U_{2}$ for $\left.s \in S-\{r\}\right\}$ where $U_{1}=U \cap\{x \in D \mid z<x\}$ and $U_{2}=\{x \in D \mid x<z\}$ if there exist $z \in D$ such that $g^{-}(b)<z<g^{-}(r)$, and if there does not exist such an element $z, \quad U_{1}=U \cap\left\{x \in D \mid g^{-}(b)<x\right\} \quad$ and $U_{2}=\left\{x \in D \mid x<g^{-}(r)\right\}$. Then $g \in V_{2}, V_{2}$ is open and $f \in V_{2}$ implies $f^{-}(r) \in U$. The other case is handled in a similar fashion.

Theorem 3. If $D$ is a topological chain and $S$ is finite, then $(M, \leqq)$ is a modular topological lattice which is nondistributive if card $S>2$ and card $D>1$.

If card $S=1, M=L$ and therefore $(M, \leqq)$ is a topological distributive lattice. If card $S \geqq 2$, then Lemma 7 and 8 establish that $\bar{V}$ is continuous since it is the composition of continuous maps. Therefore $(M, \leqq)$ is a topological lattice since $\wedge$ is continuous for every $S$. Theorem 1 establishes the modularity of $M$ while its corollary the nondistributive nature of $M$ when $3 \leqq$ card $S$ and $2 \leqq$ card $D$.

Definition. Let $n$ be a positive integer and $3 \leqq n$, then let $M_{n}$ denote the lattice constructed as the $M$ above in the case where $S=$ $\{1,2, \cdots, n\}$ and $D=\{x \in R \mid 0 \leqq x \leqq 1\}$ with its usual order and the operations of $D$ being $x \vee y=\max \{x, y\}$ and $x \wedge y=\min \{x, y\}$. When $S$ is the set of positive integers and $D$ as previously described let $M_{\infty}$ denote the lattice $M$ constructed. Then the following results are immediate.

Theorem 4. For each positive integer $n \geqq 3, M_{n}$ is a compact connected topological lattice which is modular and not distributive.

CoRollary 1. $M_{3}$ is a compact connected topological lattice, modular and not distributive, which is a two dimensional subset of $R^{3}$ that cannot be embedded in $R^{2}$.

CoRollary 2. $M_{\infty}$ is a compact connected topological semilattice, which is a modular lattice, and not a topological lattice. 


\section{REFERENCES}

1. Lee W. Anderson, On breadth and codimension of a topological lattice, Pacific J. Math. 9 (1959), 327-333.

2. Garrett Birkhoff, Lattice theory, Amer. Math. Soc. Colloquium Publications 25, New York, 1948.

3. Eldon Dyer and Allen Shields, Connectivity of topological lattices, Pacific J. Math. 9 (1959), 443-448.

Received March 11, 1968, and in revised form December 2, 1968.

University of Texas at Austin 



\section{PACIFIC JOURNAL OF MATHEMATICS}

\section{EDITORS}

H. ROYDEN
Stanford University
Stanford, California

\section{R. R. PHELPS}

University of Washington

Seattle, Washington 98105
J. DUGUNDJI

Department of Mathematics

University of Southern California

Los Angeles, California 90007

\section{RICHARD ARENS}

University of California

Los Angeles, California 90024

\section{ASSOCIATE EDITORS}

E. F. BeCKenbaCh
B. H. NEUMANN

F. WOLF
K. YoshidA

\section{SUPPORTING INSTITUTIONS}

\author{
UNIVERSITY OF BRITISH COLUMBIA \\ CALIFORNIA INSTITUTE OF TECHNOLOGY \\ UNIVERSITY OF CALIFORNIA \\ MONTANA STATE UNIVERSITY \\ UNIVERSITY OF NEVADA \\ NEW MEXICO STATE UNIVERSITY \\ OREGON STATE UNIVERSITY \\ UNIVERSITY OF OREGON \\ OSAKA UNIVERSITY \\ UNIVERSITY OF SOUTHERN CALIFORNIA
}

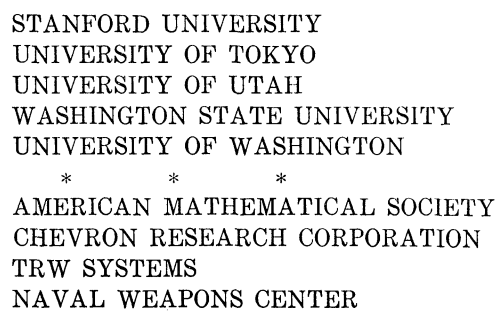

The Supporting Institutions listed above contribute to the cost of publication of this Journal, but they are not owners or publishers and have no responsibility for its content or policies.

Mathematical papers intended for publication in the Pacific Journal of Mathematics should be in typed form or offset-reproduced, double spaced with large margins. Underline Greek letters in red, German in green, and script in blue. The first paragraph or two must be capable of being used separately as a synopsis of the entire paper. It should not contain references to the bibliography. Manuscripts, in duplicate if possible, may be sent to any one of the four editors. Please classify according to the scheme of Math. Rev. 36, 1539-1546. All other communications to the editors should be addressed to the managing editor, Richard Arens, University of California, Los Angeles, California, 90024.

50 reprints are provided free for each article; additional copies may be obtained at cost in multiples of 50 .

The Pacific Journal of Mathematics is published monthly. Effective with Volume 16 the price per volume (3 numbers) is $\$ 8.00$; single issues, $\$ 3.00$. Special price for current issues to individual faculty members of supporting institutions and to individual members of the American Mathematical Society: $\$ 4.00$ per volume; single issues $\$ 1.50$. Back numbers are available.

Subscriptions, orders for back numbers, and changes of address should be sent to Pacific Journal of Mathematics, 103 Highland Boulevard, Berkeley, California, 94708.

PUBLISHED BY PACIFIC JOURNAL OF MATHEMATICS, A NON-PROFIT CORPORATION

Printed at Kokusai Bunken Insatsusha (International Academic Printing Co., Ltd.), 7-17, Fujimi 2-chome, Chiyoda-ku, Tokyo, Japan. 


\section{Pacific Journal of Mathematics \\ Vol. 29, No. $2 \quad$ June, 1969}

Bruce Langworthy Chalmers, On boundary behavior of the Bergman kernel function and related domain functionals ................... 243

William Eugene Coppage, Peirce decomposition in simple Lie-admissible power-associative rings .............................. 251

Edwin Duda, Compactness of mappings...................... 259

Earl F. Ecklund Jr., On prime divisors of the binomial coefficient......... 267

Don E. Edmondson, A modular topological lattice ............... 271

Phillip Alan Griffith, A note on a theorem of Hill ................... 279

Marcel Herzog, On finite groups with independent cyclic Sylow

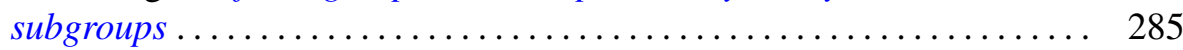

James A. Huckaba, Extensions of pseudo-valuations................. 295

S. A. Huq, Semivarieties and subfunctors of the identity functor ........ 303

I. Martin (Irving) Isaacs and Donald Steven Passman, Finite groups with small character degrees and large prime divisors. II ............ 311

Carl Kallina, A Green's function approach to perturbations of periodic

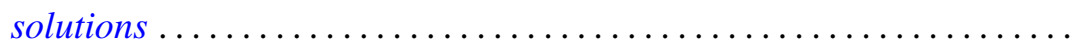

$\mathrm{Al}$ (Allen Frederick) Kelley, Jr., Analytic two-dimensional subcenter manifolds for systems with an integral ....................

Alistair H. Lachlan, Initial segments of one-one degrees ............ 351

Marion-Josephine Lim, Rank k Grassmann products ............. 367

Raymond J. McGivney and William Henry Ruckle, Multiplier algebras of

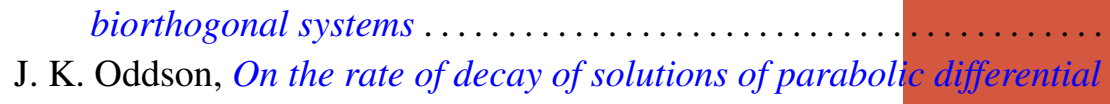

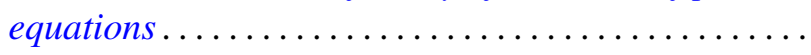

Helmut R. Salzmann, Geometries on surfaces ........... .

Annemarie Schlette, Artinian, almost abelian groups and their groups of automorphisms ............................

Edgar Lee Stout, Additional results on modules over polydisc algebras ...

Lajos Tamássy, A characteristic property of the sphere . .

Mark Lawrence Teply, Some aspects of Goldie's torsion theory. ...

Freddie Eugene Tidmore, Extremal structure of star-shaped sets ...

461

Leon Jarome Weill, Unconditional and shrinking bases in locally convex spaces... 